\title{
Influence of thin fluoropolymer film deposition on wettability of the silicon oxide nanowires array
}

\author{
Evgeniy Baranov ${ }^{1, a}$, Sergey Khmel ${ }^{1}$, Alexandr Zamchiy ${ }^{1}$, Ekaterina Kirichenko ${ }^{1}$, Aleksey Safonov ${ }^{1}$, \\ Nikolay Timoshenko ${ }^{1}$ and Veronica Sulyaeva ${ }^{2}$ \\ ${ }^{1}$ Kutateladze Institute of Thermophysics SB RAS, 630090 Novosibirsk Ac. Lavrentiev ave. 1, Russia \\ ${ }^{2}$ Nikolaev Institute of Inorganic Chemistry SB RAS, 630090 Novosibirsk Ac. Lavrentiev ave. 3, Russia
}

\begin{abstract}
In this work, we studied influence of fluoropolymer thin film deposition on wettability of the silicon oxide nanowires array. Deposition of fluoropolymer coating on the silicon oxide nanowires array changes the surface properties from hydrophilic to hydrophobic, and micro and nano scale surface roughness does not change significantly. In addition, it was shown that the deposition of fluoropolymer coating on nanowires protects the nanostructures from bundling together as a result of the attractive capillary forces that arise during evaporative drying.
\end{abstract}

\section{Introduction}

The surfaces with different wettability has wide industrial applications [1]. Recently increased interest in such surfaces in bio [2] and nano [3] technologies. Especially interesting are cases when the wetting takes limits - surface to become super hydrophilic or super hydrophobic. To change the wettability in various ways, such as nano structuring of surfaces, surface functionalization by chemical methods or by applying thin films of hydrophobic materials [4].

In this work, we studied influence of fluoropolymer thin film deposition on wettability of the silicon oxide nanowires array. If the obtained silicon oxide nanowires array were hydrophilic nature, the fluoropolymer thin film after deposition, they became hydrophobic. Untreated arrays are typically found to bundle together under surface tension forces during drying. Fluoropolymer treatment allows to avoid of undesirable liquid surface tension effects that can alter the morphology of nanowire arrays irreversibly and detrimentally [5].

\section{Experiment}

\subsection{Synthesis of the silicon oxide nanowires array}

Silicon oxide nanowires were synthesized from mixture monosilane-argon $(40 \mathrm{sccm})$ with gas diluent hydrogen $(600 \mathrm{sccm})$ by gas-jet electron beam plasma CVD method [6, 7]. Oxygen (3 sccm) was supplied directly into the vacuum chamber. The synthesis was carried out on c-Si substrate with tin film mass thickness about $60 \mathrm{~nm}$. The process of nanowire synthesis on the substrate with the catalyst

\footnotetext{
${ }^{\text {a }}$ Corresponding author : itpbaranov@gmail.com
} 
consists of three stages: heating up to operating temperature, treatment of hydrogen plasma and the actual growth of nanowires by vapor-liquid-solid (VLS) mechanism. The synthesis temperature was $335^{\circ} \mathrm{C}$.

\subsection{Deposition of thin fluoropolymer film}

In this study, the thin fluoropolymer coating is deposited by the HWCVD method [8-10]. Hexafluoropropylene oxide $\left(\mathrm{C}_{3} \mathrm{~F}_{6} \mathrm{O}\right)$ was used as the precursor gas of the fluoropolymer coating. The cooled substrate holder with the diameter of $90 \mathrm{~mm}$ was in the center of the chamber, and substrate with the silicon oxide nanowires array was fixed there. Above the substrates at a distance of $50 \mathrm{~mm}$, there is the catalytic activator in the form of a mesh with pitch of $8 \mathrm{~mm}$. The temperature of the wire was constant. The precursor gas pressure in the deposition chamber was 0.5 Torr. The precursor gas flow rate is $100 \mathrm{sccm}$. The temperature of activating wire mesh is $580^{\circ} \mathrm{C}$, the target temperature was about $30^{\circ} \mathrm{C}$. The thickness of the fluoropolymer film is about $100 \mathrm{~nm}$.

\subsection{Measuring techniques}

The surface morphology was determined by the methods of scanning electron microscopy (SEM) using the JEOL JSM-6700F microscope.

The contact angle of the sample with water was measured by DSA-100 KRUSS device with highprecision system of liquid supply with a minimal dosing step of $0.1 \mathrm{mcl}$. optic system, and diffusion halogen light source $(50 \mathrm{~W})$.

\section{Results and discussion}

SEM image on Fig. 1a shows of the silicon oxide nanowires array. Each microrope consists of numerous nanowires with average diameter about $15 \mathrm{~nm}$. Nanowires bunch is on the bottom of the catalyst particle, whereas the nanowires are absent at the top of it.

Analysis of the photograph (Fig. 1b) of the water droplet on the silicon oxide nanowires grown on c-Si substrate shows the measured contact angle was $<20^{\circ}$. This confirms that the surface of the silicon oxide nanowires array, synthesized in this work, is hydrophilic in nature. The measured contact angle was different for the left and right side of the drop. We can explain this phenomenon by the fact that microropes are not perpendicular to the surface, and have a different angle of inclination. In addition, it was observed that the droplets on the surface have a very large imbibition layer [11]. We assume that water penetrate easily into grooves constituting the relief in case of Cassie impregnating wetting [12]. SEM image of the silicon oxide nanowires array after exposure to water is shown on Fig. 1c. It is evident that microropes stuck together in bundles of several ones. We assume that this is the influence of the water capillary forces that arise during evaporation process from the nanostructured surface.

SEM image on Fig. 1d shows of the silicon oxide nanowires array covered with the thin fluoropolymer film. The surface of the microropes is covered with a thin layer of the film, so it is not visible the structure of individual nanowires. Herewith on the top of microrope, on the catalyst particle, structure with high roughness can be observed.

The wettability of the surface of the silicon oxide nanowires array covered with the thin fluoropolymer film is shown on Fig 1e. The observed static water contact angle was $>107^{\circ}$. This confirms that the surface of the silicon oxide nanowires array covered with the thin fluoropolymer film is hydrophobic in nature. This means that the covering with the thin fluoropolymer film has significant impact on the properties of the surface such as wettability. In this case, the measured contact angle was approximately equal for the left and right side of the drop. 


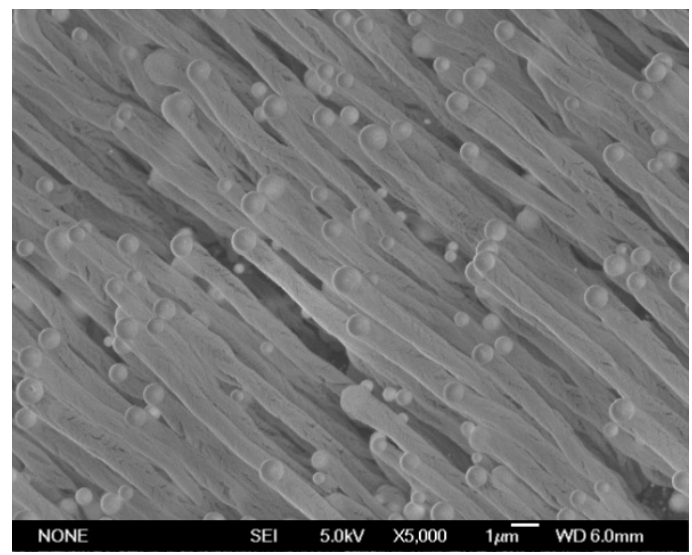

a

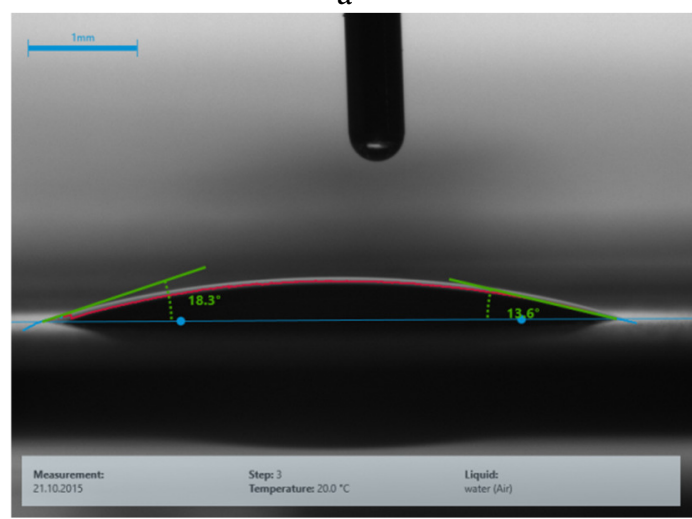

b

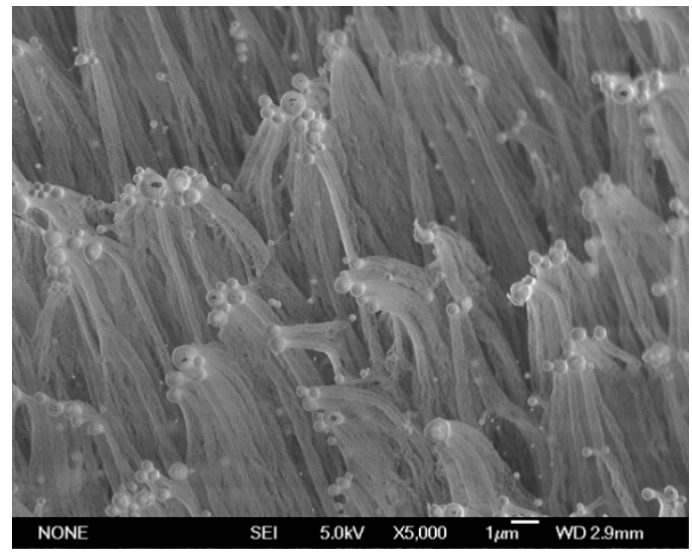

$\mathrm{c}$

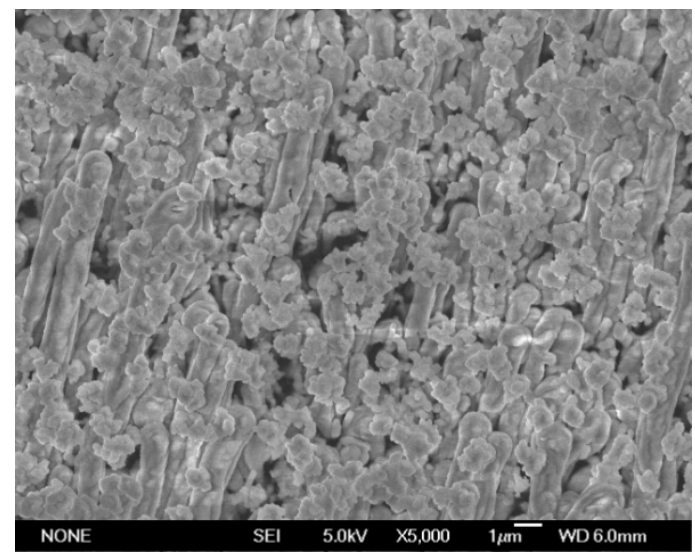

$\mathrm{d}$
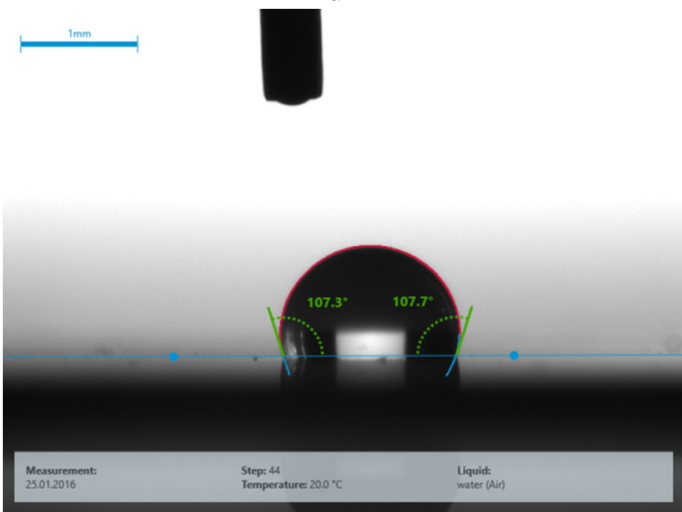

e

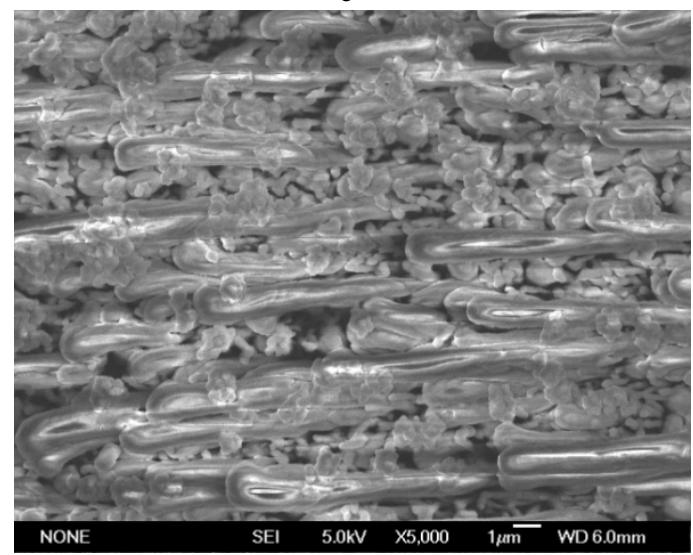

$\mathrm{f}$

Figure 1. $a$ - SEM image of the silicon oxide nanowires array: $b$ - the photograph of the water droplet on the silicon oxide nanowires; $c$ - SEM image of the silicon oxide nanowires array after exposure to water; $d$ - SEM image of the silicon oxide nanowires array covered with the thin fluoropolymer film; e - the photograph of the water droplet on the silicon oxide nanowires array covered with the thin fluoropolymer film; $f$ - SEM image of the silicon oxide nanowires array covered with the thin fluoropolymer film after exposure to water.

SEM image of the silicon oxide nanowires array covered with the thin fluoropolymer film after exposure to water is shown on Fig. 1f. Obtaining of the clear picture of the structure with help of the electron microscope is a big problem, because of strong charging of the surface. But it is safe to say that 
microropes didn't stuck in bunches after water evaporation. The thin fluoropolymer film deposition of the silicon oxide nanowires array shows protective properties for the nanostructured surface.

\section{Conclusion}

In this work, we studied influence of fluoropolymer thin film deposition on wettability of the silicon oxide nanowires array. If the obtained silicon oxide nanowires array were hydrophilic nature, the fluoropolymer thin film after deposition, they became hydrophobic. In addition, it was shown that the coating of polymer nanowires protects the nanostructure from water.

\section{Acknowledgements}

The work was supported by the grant of the President of the Russian Federation for young scientists MK 5052.2016.08 (deposition of thin fluoropolymer film) and by the grant of the Russian Science Foundation \#15-19-30038 (synthesis of the silicon oxide nanowires array).

\section{References}

1. N. Verplanck, Y. Coffinier, V. Thomy, R. Boukherroub, Nanoscale Res. Lett., 2, 577 (2007)

2. G. Piret, Y. Coffinier, C. Roux, O. Melnyk, R. Boukherroub, Langmuir, 24, 1670 (2008)

3. C. Kuo, C. Gau, Journal of The Electrochemical Society, 157, K201 (2010)

4. L. B. Boinovich, A. M. Emelyanenko, Russ. Chem. Rev., 77, 583 (2008)

5. K. Lau, J. Bico, K. Teo, M. Chhowalla, G. Amaratunga, W. Milne, G. McKinley, K. Gleason, Nano Lett., 3, 1702 (2003)

6. A. Zamchiy, E. Baranov, S. Khmel, Physica status solidi (c), 11, 1397 (2014)

7. E. Baranov, A. Zamchiy, and S. Khmel, Technical Physics Letters, 39, 1023 (2013)

8. K. Lau, H. Lewis, S. Limb, M. Kwan, K. Gleason, Thin Solid Films, 395, 288 (2001)

9. K. Kenneth, Y. Mao, H. Lewis, S. Murthy, B. Olsen, L. Loo, K. Gleason, Thin Solid Films, 501, 211 (2006)

10. A. Rebrov, A. Safonov, N. Timoshenko, Technical Physics Letters, 35, 395 (2009)

11. N. Dhillon, J. Buongiorno, K. Varanasi, Nat. Commun. 6, 8247 (2015)

12. A. Mohamed, A. Abdullah, N. Younan, Arabian Journal of Chemistry, 8, 749 (2015) 